\title{
SUR UNE NOUVELLE FORME DE GLYCOSURIE CHEZ LES GRENOUILLES,
}

PAR

\author{
St-J. Przylecki (Varsodie).
}

J'Al observé une glycosurie chez les grenouilles transportées brus$\int$ quement de la température de $0^{\circ}$ à $20-25^{\circ}$. L'albuminurie est dans ces conditions un phénomène assez rare.

Je me suis proposé, dans ce travail, d'étudier les conditions d'apparition du sucre dans l'urine.

Le tableau I montre la marche du phénomène.

Déjà après 12 heures de séjour à la température de $25^{\circ}$, la réaction de la liqueur Fehling est nettement positive elle reste bien nette après 24,36 et 48 heures. Après 60 heures, on ne l'observe que rarement et après 72 , aucune grenouille ne présente plus de sucre dans l'urine.

La glycosurie apparaît chez les animaux maintenus dans l'eau, ainsi que dans la chambre humide. Ce n'est donc pas l'augmentation de la quantité d'urine sécrétée qui provoque ce phénomène, d'autant plus que nous ne le voyons pas chez les animaux maintenus préalablement pendant 4 à 5 jours dans la chambre humide (1-2 cc. d'urine par $100 \mathrm{gr}$. et $24 \mathrm{~h}$.) et ensuite complètement immergés dans de l'eau à la température de $22^{\circ}(25-35 \mathrm{cc}$. d'urine par $100 \mathrm{gr}$. de tissus et 24 h.).

Les grenouilles maintenues à la température de $0^{\circ}$ ne présentaient pas de sucre dans l'urine. Il n'apparaissait qu'après l'élévation de la température. Ce fait prouve que la glycosurie décrite n'est pas identique au phénomène de "Kältediabetes" observé par LoEwit.

Nous pouvons donc admettre que la cause de la glycosurie est une augmentation brusque de la température.

Le changement de température doit être assez grand pour provoquer le phénomène. En déplaçant les grenouilles de $0^{\circ}$ à $10^{\circ}$ on n'obtient aucun effet.

La quantité de sucre contenu dans l'urine (') était assez grande,

(') Les animaux avaient l'anus ligaturé. Après un certain temps, je recueillais l'urine et déterminais le sucre par la méthode de MichaElis. 


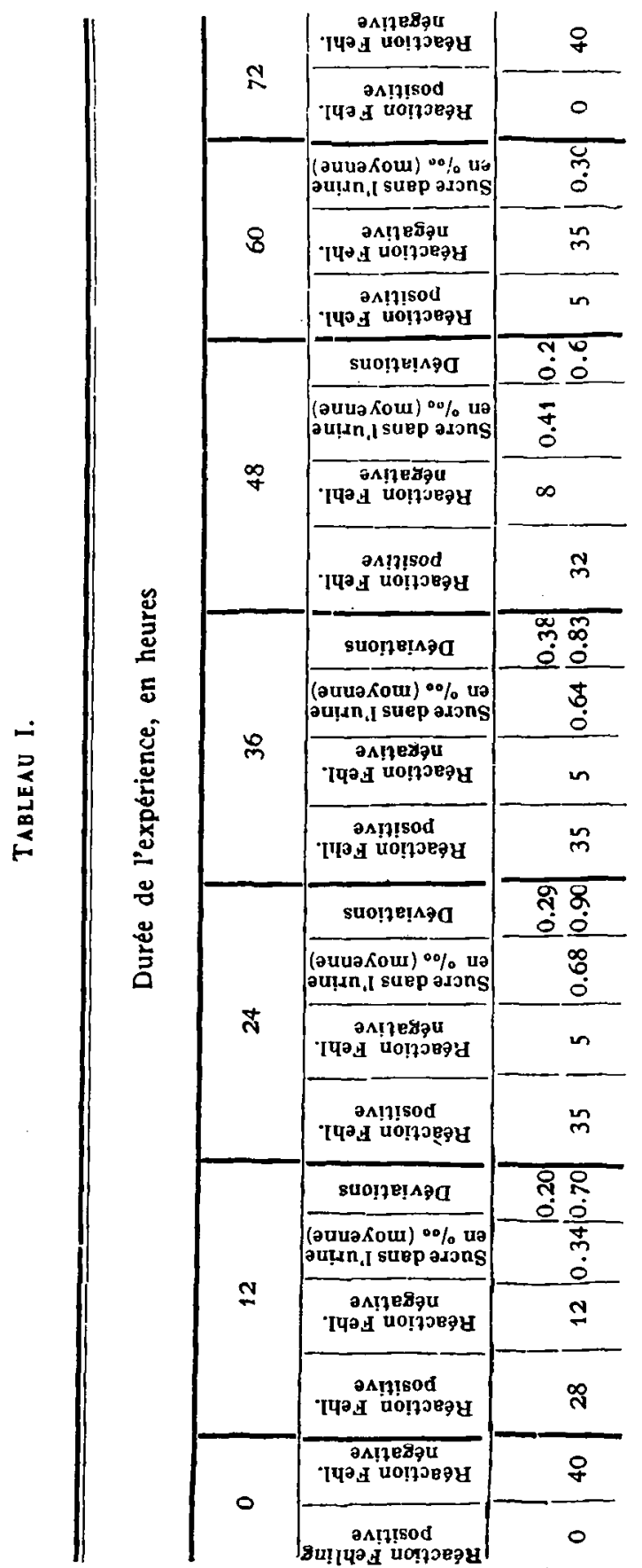

mais ne dépassait jamais 1 mgr. par cc. J'ai trouvé en moyenne $0.68 \mathrm{mgr}$.

En admettant qu'une grenouille de 100 gr. excrétait pendant $24 \mathrm{~h}$. environ 30 cc. d'urine, la quantité de sucre excrétée par 100 gr.et 24 h.est à peu près de $30 \mathrm{mgr}$. Il reste à élucider la cause de la glycosurie.

Comme nous le savons, l'apparition du sucre dans l'urine est le plus souvent provoquée par une hyperglycémie. Envisageant les faits connus, à savoir que les grenouilles maintenues à différentes températures renferment dans le sang des quantités différentes de sucre (BANG trouve $0.4 \%$ à la température normale et $0.2 \%$ a celle de la cave), il m'a paru vraisemblable que la glycosurie étudiée par moi était provoquée par une augmentation rapide du sucre dans le sang. 
Pour vérifier cette hypothèse, j'ai déterminé la teneur en sucre du sang de grenouilles, conservées pendant 20 à 30 heures à $20-22^{\circ}$, d'après la méthode Michaelis, modifiée par LESSER ; et j'ai trouvé une quantité de sucre atteignant $0.67 \%$ (tableau II).

Nous voyons donc que la glycosurie, provoquée par le changement brusque de la température, est due a une augmentation du sucre contenu dans le sang.

TABLEAU II. - Sucre contenu dans le sang en $\%$.

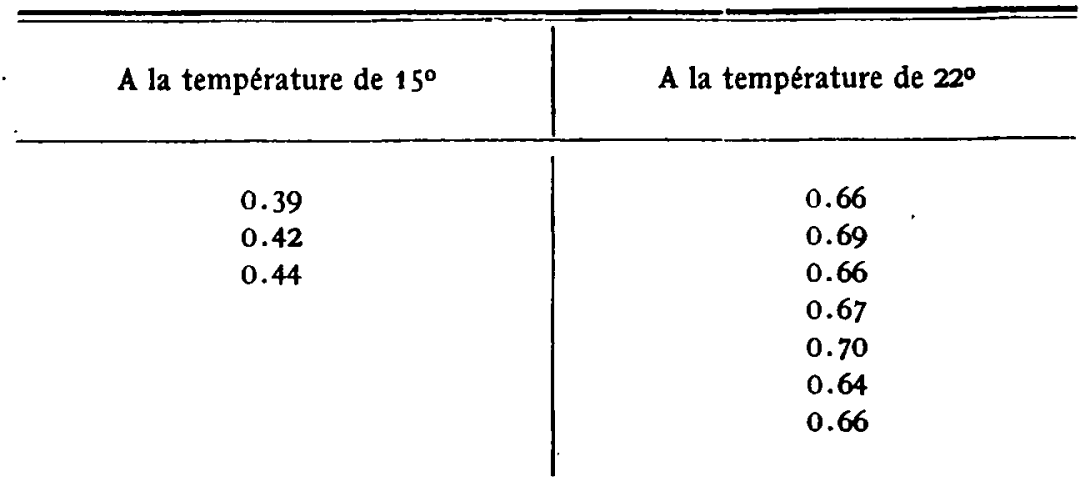

BIBLIOGRAPHIE

M. LOEwIT. - Arch. f. Exper. Pathol. u. Pharmak., 1909, vol. 60 et 1910, vol. 62.

L. Micha elis. - Bioch. Zeitschr., 1913, vol. 59.

I. BANG. - Der Blutzucker. Wiesbaden, 1913.

E. LESSER. - Bioch. Zeitschr., 1913, vol. 54. 